\begin{tabular}{|l|l|}
$\begin{array}{l}\text { Postprint } \\
\text { Version }\end{array}$ & 1.0 \\
\hline Journal website & $\underline{\text { http://www.sciencedirect.com/science/article/pii/S0277953614004717 }}$ \\
\hline Pubmed link & $\underline{\text { http://www.ncbi.nlm.nih.gov/pubmed/25063966 }}$ \\
\hline DOI & $10.1016 /$ j.socscimed.2014.07.042 \\
\hline
\end{tabular}

This is a NIVEL certified Post Print, more info at http://www.nivel.eu

\title{
Negotiating jurisdiction in the workplace: A multiple-case study of nurse prescribing in hospital settings
}

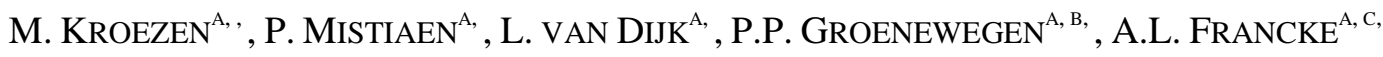 \\ ${ }^{a}$ NIVEL, Netherlands Institute for Health Services Research, PO Box 1568, 3500 BN \\ Utrecht, The Netherlands \\ ${ }^{\mathrm{b}}$ Department of Sociology and Department of Human Geography, Utrecht University, PO \\ Box 80115, 3508 TC Utrecht, The Netherlands \\ ${ }^{c}$ Department of Public and Occupational Health, EMGO Institute for Health and Care \\ Research (EMGO+) of VU University Medical Center, Van der Boechorststraat 7, 1081 BT \\ Amsterdam, The Netherlands
}

\begin{abstract}
This paper reports on a multiple-case study of prescribing by nurse specialists in Dutch hospital settings. Most analyses of interprofessional negotiations over professional boundaries take a macro sociological approach and ignore workplace jurisdictions. Yet boundary blurring takes place and healthcare professionals renegotiate formal policies in the workplace. This paper studies the division of jurisdictional control over prescribing between nurse specialists and medical specialists in the workplace, and examines the relationship between workplace jurisdiction and legal jurisdiction over prescribing. Data collection took place in the Netherlands during the first half of 2013. The study used indepth interviews with fifteen nurse specialists and fourteen medical specialists, non-participant observation of nurse specialists' prescribing consultations and document analysis. Great variety was found in the extent to which and way in which nurse specialists' legal prescriptive authority had been implemented. These findings suggest that there is considerable discrepancy between the division of jurisdictional control over prescribing at the macro (legal) level and the division at the micro (workplace) level.
\end{abstract}

\section{INTRODUCTION}

Governments increasingly see the shifting of tasks from physicians to nurses as a suitable policy response to current problems in healthcare, such as the shortage of 
Kroezen, M., Mistiaen, P., Dijk, L. van, Groenewegen, P.P., Francke, A.L. Negotiating jurisdiction in the workplace: a multiple-case study of nurse prescribing in hospital settings. Social Science \& Medicine: 2014, 117(sept), 107-115

physicians and rising costs (Buchan and Dal Poz, 2002, Horrocks et al., 2002, Lewis, 2001, Raad voor de Volksgezondheid en Zorg, 2002 and Wanless, 2002). At the same time, the nursing profession is attempting to increase its professional status, using several strategies for occupational advancement (Gerrish et al., 2003). These joint developments have resulted in nurses taking up new positions - such as the role of clinical nurse specialist in the United Kingdom (Courtenay and Carey, 2009) and nurse specialist in the Netherlands (Van der Peet, 2010 and Van Meersbergen, 2011) - and new tasks, one of which is the prescribing of medicines (Kroezen et al., 2012a and Kroezen et al., 2012b). In the Netherlands, nurse specialists work autonomously and make independent diagnoses and treatment decisions (see Box 1). Since January 2012, they have been legally allowed to prescribe medicines and have shared legal jurisdiction over prescribing with physicians. While medical associations initially showed reservations or even reluctance towards the introduction of nurse prescribing (Kroezen et al., 2012a and Kroezen et al., 2012b), their resistance gradually decreased and they instead cooperated with nursing associations at the legal level whilst trying to influence the arrangement of nurse prescribing in such a way that the outcomes would be as beneficial as possible for themselves (Kroezen et al., 2013a and Kroezen et al., 2013b).

\section{[Box 1]}

When nurses take up new positions or take over tasks from physicians, professional boundaries are shifted, and the division of jurisdictional control between the medical and nursing profession is changed. Up to now, little is known about how nurse prescribing takes shape in everyday healthcare practice. In this paper, we examine the division of jurisdictional control over prescribing between nurse specialists (with a Master's degree in Advanced Nursing Practice) and physicians in the workplace, and study the extent to which workplace jurisdiction over prescribing resembles legal jurisdiction over prescribing. In other words, we examine the extent to which nurse specialists' legal prescriptive authority resembles the way in which they are currently prescribing in everyday healthcare practice and what role medical specialists play in the prescribing process.

\section{Jurisdiction in the system of professions}

Because prescribing has traditionally been the sole domain of the medical profession (Buckley et al., 2006, Fisher, 2010 and Goundrey-Smith, 2008), the expansion of prescriptive authority to include nurse specialists touches on issues of professional domains and competition between professions for jurisdiction over tasks. Jurisdiction is crucial for professionals because it is their means of continued livelihood (Bechky, 2003). Professionals who are recognised as experts in a certain area, in this case the area of prescribing medicines, typically possess a form of cultural capital whose ownership confers status and power (Mclaughlin and Webster, 1998 and Petrakaki et al., 2012). Therefore Abbott (1988) labels jurisdiction - "the link between a profession and its work" - as the central phenomenon of professional life. Jurisdiction, in this sense, can be understood as professional control over the work itself and the knowledge mobilised within the occupation.

Since one profession can pre-empt another's jurisdiction or control over a task, professions exist in an interdependent system with competing jurisdictional claims. According to Abbott (1988), professions can claim jurisdictional control over tasks in several arenas, namely the legal arena, the workplace arena and the arena of public 
opinion. The particular arena in which jurisdictional negotiations take place shapes the form that they assume (Allen, 2000 and Mizrachi and Shuval, 2005). In this paper, our focus will be on the workplace arena and the legal arena, and the relationship between these two.

Professional competition regarding jurisdiction over a task can have various outcomes. After all, not every profession striving for full jurisdiction will obtain it. Most professional conflicts over jurisdiction result in what are termed "limited jurisdictional settlements” (Abbott, 1988). These are alternatives to the situation in which one or more professions hold full jurisdiction over a task. In a jurisdictional settlement, professions share the jurisdiction over a task, whereby control is distributed between the professions to a greater or lesser extent equally, depending on the type of jurisdictional settlement concerned. Abbott (1988) discerns several types of jurisdictional settlement, including subordination, whereby an incumbent profession controls the division of labour for one or more subordinate groups, intellectual jurisdiction, in which the incumbent profession controls the cognitive knowledge of an area but allows practice by other professions and client differentiation, in which different segments of a profession serve different client groups.

In the Netherlands, nurse specialists' legal prescriptive authority is comparable to that of physicians. Both physicians and nurse specialists are allowed to independently prescribe any licensed medicine for any medical condition within their specialism and competence (see Box 1). However, it should be noted that physicians have a significantly wider field of competence. Nonetheless, in the legal arena, nurse specialists and physicians share full jurisdiction over prescribing. In general, however, formalised jurisdictions have a rather vague relation to professional workplace realities (Abbott, 1988). In the workplace, professional boundaries cannot be strictly maintained and healthcare professionals renegotiate formal policies (Sanders and Harrison, 2008 and Spilsbury and Meyer, 2004). Allen (1997) for example showed how boundary blurring took place between doctors and nurses on a surgical and medical ward in a general hospital, and Snelgrove and Hughes (2000) likewise demonstrated the role blurring and informal crossing of boundaries that takes place between doctors and nurses. Hence, features of the work setting mediate the formal division of labour (Allen, 2000). Yet investigations of workplace occupational boundaries are rare. Most analyses of inter-occupational competition take a macrosociological approach, looking at the level of the professional field rather than the organisational level where interactions between professionals take place on a daily basis (Bechky, 2003). This is problematic, as organizations and individuals can mediate the influence of legislation on professional work jurisdictions and roles, and influence the extent to which shifts in professional boundaries take place in practice, for example by not formally recognising new sets of knowledge and skills in definitions of work roles and expertise, through training or in regulations (Currie et al., 2010). Some of the rare studies that have looked into the issue of enacted professional jurisdictions (e.g. Barley, 1986, Barrett et al., 2012, Currie et al., 2012 and Salhani and Coulter, 2009) draw attention to the fact that purposive yet subtle actions of individuals and organisations, such as day-to-day adjustments, adaptations and compromises, can substantially change the division of jurisdiction on the work floor. The present study contributes to the literature by explicitly examining the link between the macro- and micro level by taking into 
Kroezen, M., Mistiaen, P., Dijk, L. van, Groenewegen, P.P., Francke, A.L. Negotiating jurisdiction in the workplace: a multiple-case study of nurse prescribing in hospital settings. Social Science \& Medicine: 2014, 117(sept), 107-115

consideration the legal arena and the workplace arena, and the relationship between these two.

This study has a twofold aim: first, to investigate the division of jurisdictional control over prescribing between Dutch nurse specialists (with a Master's degree in Advanced Nursing Practice) and physicians in the workplace; second, to study the extent to which workplace jurisdiction over prescribing resembles legal jurisdiction over prescribing. The following research questions were addressed:

1. How does prescribing by nurse specialists take shape in the workplace?

2. How is jurisdictional control over prescribing divided between nurse specialists and physicians in the workplace?

3. To what extent does workplace jurisdiction over prescribing resemble legal jurisdiction over prescribing?

\section{THE STUDY}

\subsection{Research approach}

As prescribing processes are complex and context dependent, a multiple-case study research strategy was adopted (Green and Thorogood, 2004). Cases were defined as nurse specialists working in hospital settings and prescribing medicines to patients. Data on nurse specialists' prescribing practices were collected using a multi-method approach consisting of semi-structured interviews with nurse specialists and medical specialists, observations of nurse specialists' prescribing consultations and document analysis. In line with earlier studies on enacted professional jurisdictions (Barley, 1986, Barrett et al., 2012, Currie et al., 2012 and Salhani and Coulter, 2009), we chose to employ a variety of research methods to allow for data triangulation. In doing so, we sought to increase confidence in the validity of our findings by integrating and synthesizing different sources of evidence (Currie et al., 2012 and Green and Thorogood, 2004). This enabled us, for example, to 'validate' accounts of behaviour from interview data. After all, interview accounts cannot always be read as straightforward descriptions of practices (Allen, 1996).

\subsection{The sample of nurse specialists and physicians}

In this study, we aimed to select nurse specialists representing a range of work settings (university hospital versus general hospital), clinical contexts and nurse specialties. We therefore used purposive sampling. The aim of purposive sampling is to select participants who will generate appropriate data (Green and Thorogood, 2004 and Polit and Beck, 2004). We conveniently selected three university hospitals and two general hospitals in the Netherlands. Within the hospitals, we purposively selected three nurse specialists working in different specialisms. However, some of the selected nurse specialists said they were not yet prescribing medicines, for example because they had not yet obtained permission from the hospital board of directors, and we therefore had to include other nurse specialists. It should be noted that permission from the board of directors is not legally required for nurse specialists to prescribe, but most hospitals nevertheless apply this rule. As the number of prescribing nurse specialists turned out to be fairly low in some hospitals, we eventually had to select some nurse specialists working in the same specialism and/or on the same ward.

All fifteen selected nurse specialists received an introductory letter about the study by email and were asked whether they would be interested in participating. If they 
said yes, a first appointment with one of the researchers was scheduled during which more information about the study was provided and all remaining questions that the nurse specialist might have were answered. All the invited nurse specialists agreed to participate and the final study sample consisted of fifteen nurse specialists. Because we aimed to include those medical specialists with whom the nurse specialists collaborated most often in their daily practice, the decision as to which medical specialist would be best suited to participate in the study was left up to the nurse specialists. Fourteen of the fifteen medical specialists who were nominated by the nurse specialists to participate in our study agreed to be interviewed. Table 1 shows the different specialisms in which the participating nurse specialists and medical specialists were working.

\section{[TABLE 1]}

\section{DATA COLLECTION}

Data collection took place during the first half of 2013, more than a year after nurse specialists in the Netherlands had obtained legal prescriptive authority. To study how prescribing by nurse specialists is taking shape in the workplace and how jurisdictional control over prescribing is divided between nurse specialists and physicians, we performed non-participant observations of nurse specialists' consultations, conducted semi-structured interviews with nurse specialists and medical specialists, and performed document analysis. On the basis of these analyses, we subsequently studied the extent to which nurse specialists' workplace jurisdiction over prescribing resembles their legal jurisdiction over prescribing. As we did not aim to compare nurse specialist and physician prescribing, physicians' prescribing consultations were not part of our data collection.

\subsection{Semi-structured interviews with nurse specialists and physicians}

Interviews provided the primary source of data. The interviews with nurse specialists and medical specialists were semi-structured and were guided by topic lists that were compiled on the basis of the literature and previous studies by the research group (Kroezen et al., 2011, Kroezen et al., 2012a, Kroezen et al., 2012b, Kroezen et al., 2013a and Kroezen et al., 2013b). The interview topics were formulated after examining the relevant literature and preliminary observations of nurse specialist prescribing (see Box 2). The majority of interviews were conducted face to face by a member of the research team who was trained in qualitative interviewing techniques. However, for various reasons, three interviews with medical specialists were conducted by phone. All interviews were audiotaped and transcribed verbatim, and a copy of the transcript was sent to participants for alterations, additional comments and approval. The approved interview transcripts formed the basis for analysis and were imported into the data analysis software package MAXQDA 2007 for thematic analysis (MAXQDA, 2007).

\section{[Box 2]}

\subsection{Non-participant observation of nurse specialists' consultations}

Non-participant observation of nurse specialists' prescribing consultations provided information that was used to supplement data collected through interviews. The consultations took place in both outpatient and inpatient hospital settings. We aimed 
to observe at least three consultations with each nurse specialist in which a

prescription was issued by the nurse specialist. We reached this target with twelve of the fifteen nurse specialists. Because we observed more than the minimum of three prescribing consultations with five nurse specialists, our total number of observed prescribing consultations is 49 . The number of consultations needed to collect three prescribing consultations varied from three to nineteen consultations among the twelve nurse specialists.

Data on nurse specialists' prescribing practices were collected by a member of the research team using a structured observation checklist. Moreover, for each prescribing consultation, details of the prescription (including dose and directions, patient age, gender, presenting condition, diagnosis, comorbidity and other medications taken) were recorded and/or extracted from patient records.

\subsection{Document analysis}

Additionally, we collected all documents that were used by nurse specialists in the prescribing process. These included individual prescribing agreements with hospital pharmacies, ward-based formularies and references to online directories, such as the Pharmacotherapeutic Compass (Farmacotherapeutisch Kompas in Dutch).

\subsection{Ethical considerations}

Our study design was send to the Medical Ethical Committee of the VU University Medical Center and judged as not being subject to the Medical Research Involving Human Subjects Act. Therefore, no formal medical ethics review was required. The study design was reviewed and approved by some of the local institutional review boards of the participating hospitals. Personal data were handled confidentially and processed anonymously, as required by the rules of the Dutch Data Protection Act (Wet bescherming persoonsgegevens in Dutch) and the applicable codes of conduct for scientific researchers.

The study aims, data collection methods, procedures and the confidential, voluntary and anonymous nature of the study were explained to all participating nurse specialists and physicians by a member of the research team. Prior to the nonparticipant observation of consultations, details of the study and its voluntary nature were briefly explained to patients by a member of the research team and by an introductory letter. All patients gave their informed - written - consent to their participation in the study.

\section{DATA ANALYSIS}

We performed a thematic analysis of the interview transcripts, observation reports and documents gathered through the document analysis to answer our research questions (Green and Thorogood, 2004). Data analysis began at an early stage in the research in order to be able to introduce any necessary changes in the topic lists for the interviews and checklist for the observations. Data were analysed both inductively and deductively. We searched the data for concepts that were directly linked to jurisdictional control, interprofessional collaboration, interprofessional tensions, and nurse specialists' prescribing practices and considerations. Additionally, data were analysed inductively and compared for common statements and claims. To ensure the reliability and validity of coding, two researchers (MK and PM) coded each interview transcript and observation report independently and verified each other's work. Discrepancies in coding were discussed until agreement 
Kroezen, M., Mistiaen, P., Dijk, L. van, Groenewegen, P.P., Francke, A.L. Negotiating jurisdiction in the workplace: a multiple-case study of nurse prescribing in hospital settings. Social Science

\& Medicine: 2014, 117(sept), 107-115

was reached that the data in the transcripts were accurately represented by the revised themes. Recurring themes were identified and classified, and text fragments were sorted according to the thematic framework.

\section{FINDINGS}

Findings are organised around the main themes found through data analysis, i.e.: nurse specialists' prescribing practices, protocols, formularies and guidelines used whilst prescribing, cooperation between nurse specialists and medical specialists when prescribing medicines, and difficulties surrounding nurse specialists' prescriptive authority. Quotations were chosen to illustrate the themes.

\subsection{Nurse specialists' prescribing practices}

We found great variety in nurse specialists' prescribing practices, both in terms of the degree of prescribing and in terms of the types of prescriptions. The extent to which nurse specialists made use of their prescriptive authority in everyday practice varied considerably. Some nurse specialists said they prescribed "lots of prescriptions", for up to sixteen patients a day, while others estimated they wrote out a prescription three to ten times a week on average, and a small group stated they prescribed medication only once a week. These mixed results are in accordance with our observations, for example the number of consultations needed to obtain three prescribing consultations varied among the nurse specialists from three to nineteen consultations. Moreover, for one nurse specialist we were able to observe 28 prescribed medicines, while for others we were unable to reach our target of three prescribing consultations.

The same variety could be observed with regard to the type of prescription and the range of medicines that nurse specialists were allowed to prescribe in their work setting and the extent to which this was delimited at the hospital, ward or individual level. While most nurse specialists were allowed to independently prescribe both initial and repeat prescriptions, some were required to check their initial prescriptions with their medical specialist, except for medication that both considered as having a relatively low risk. Moreover, in one of the observed cases, the nurse specialist was not allowed to sign initial prescriptions; the medical specialist signed a prescription that was filled in by the nurse specialist.

Most nurse specialists were allowed to prescribe a defined, often relatively limited, number of medicines as set out in protocols or (personal) formularies. However, some nurse specialists had considerably more freedom and could prescribe a broader range of medicines than others, depending on the protocols and formularies that were used in their workplace. In general, nurse specialists and medical specialists were satisfied with the range of medicines that could be prescribed by nurse specialists. Some physicians referred to these medicines as "common-or-garden medicines", while others noted that the range of medicines should not be too restricted as patients do not fit into neat compartments and this would create an unworkable situation.

\subsection{Protocols, guidelines and formularies used whilst prescribing}

The number of different protocols, guidelines and formularies that were used by nurse specialists' whilst prescribing was extensive. Moreover, the level of applicability of these documents differed hugely, ranging from guidelines drafted by international professional associations to individual formularies developed by the individual nurse specialist. Most nurse specialists used various different protocols, 
guidelines and formularies in their everyday practice. In general, most nurse

specialists appeared to prescribe on the basis of either local hospital protocols or ward-based protocols, used by all prescribers in the hospital or ward, or on the basis of local protocols specifically designed for nurse specialists:

"Actually, most medication has already been described in an allogeneic transplant protocol; what is appropriate to start with in certain cases, what I said about the preventive medication, that's all been captured in a protocol.” (Nurse specialist 2) "Yes, those [protocols for nurse specialists] are tailor-made. With a proposal by the nurse specialists themselves, which medicines they encounter and where they feel competent and skilled. And that has been approved in the department meeting." (Medical specialist 8)

As indicated by the above quote, where nurse specialists prescribed on the basis of protocols that were specifically designed for them, these protocols were almost always developed and/or approved by medical staff, and in most cases also by the hospital pharmacist.

In some hospitals, there were also formal arrangements about the kind of medicines for which nurse specialists were required to consult a medical specialist before prescribing them. Most of the time these included antibiotics and medicines with a relatively high risk, such as immunosuppressives.

\subsection{Cooperation between nurse specialists and medical specialists when prescribing medicines}

There was considerable consultation between nurse specialists and physicians when nurse specialists prescribed, or considered prescribing, medicines. If nurse specialists had the slightest doubt about whether to prescribe, what to prescribe or which dose to prescribe, they contacted the medical specialist:

"If I feel just a little bit unsure, I just get a doctor here.” (Nurse specialist 12)

"If something is being prescribed, there is always consultation with me, like: 'This patient has this and that, and now I see this and I wanted to prescribe that. What do you think?” (Medical specialist 3)

Some nurse specialists also said that consultation with a physician always takes place in the case of the prescription of new medicines or deviations from standard protocols. This was reflected in our observations, in which, for example, one nurse specialist asked the collaborating medical specialist for advice about which type of antibiotic to prescribe, and another nurse specialists postponed the prescription of a non-standard medicine until she had discussed it with the medical specialist.

Moreover, some nurse specialists were not allowed to treat medically complex patients; these patients were treated solely by the medical specialist.

The consultations between nurse specialists and medical specialists about prescribing decisions were almost exclusively informal in nature. Nurse specialists either walked round to the medical specialist and discussed the matter with them on the spot before returning to their patient, or they asked medical specialists to drop by during their patient consultation. Nurse specialists also phoned and emailed physicians for advice and additional checks on their prescriptions:

"I pop round to someone or I call someone. And we're not going to make an appointment for that first, that doesn't work.” (Nurse specialist 1)

The high frequency and informal nature of prescribing consultations between nurse specialists and physicians was confirmed by our observations. In more than a quarter of the prescribing consultations that we observed, nurse specialists contacted their 
Kroezen, M., Mistiaen, P., Dijk, L. van, Groenewegen, P.P., Francke, A.L. Negotiating jurisdiction in the workplace: a multiple-case study of nurse prescribing in hospital settings. Social Science

\& Medicine: 2014, 117(sept), 107-115

medical specialist to discuss a possible prescription. These consultations were always informal in nature and included dropping in on the physician for advice, asking the physician to come in during a patient consultation, and jointly visiting the nurse specialist's patient and discussing medication issues at the patient's bedside. The large majority of nurse specialists and medical specialists said they liked working in this way. Almost all participants experienced an increase in efficiency in their everyday practice. Before nurse specialists obtained prescriptive authority, all medicines were prescribed by medical specialists. Even though nurse specialists often already did the preparatory work and filled in the prescription, it still needed to be signed by a medical specialist, causing unnecessary delays for both healthcare professionals and patients. One medical specialist noted that "it makes your organisation more flexible" (Medical specialist 4) and a nurse specialist explained: "It is easier for me, because now you can finish everything with the patient and you don't have to wait in the corridor for a quarter of an hour until the doctor has signed the prescription." (Nurse specialist 4)

However, while in general the prescribing process may have become more efficient, our observations showed that in cases where the nurse specialist consulted with the medical specialist before prescribing, in more than a quarter of the prescribing consultations, there was still considerable waiting time involved on the part of the nurse specialist and patient.

In most nurse specialist-physician partnerships, there was room for mutual constructive criticism and nurse specialists felt they had an equal relationship with physicians. As one nurse specialist expressed it:

"And nowadays, I must say, it actually takes place on a very equal footing. We can discuss things with each other, we can talk about things. And ultimately the surgeon is the boss, that's clear. But by now we have enough credibility so that, when I have good arguments, they listen to me.” (Nurse specialist 13)

It appears that the foundation for the generally good cooperation between nurse specialists and medical specialists as regards prescribing lies in the mutual trust that has been built up between them over the years. Often, the nurse specialists and medical specialists had been cooperating for years already and knew each other quite well before nurse specialists' prescriptive authority was introduced. This made the change easier. Moreover, many of the medical specialists played a role in the nurse specialists' training, so they were well aware of how much training the nurse specialist had received and of what quality.

“(...) that they know us, that makes a difference as well. We have been working together in a team for so long that they know your capabilities and you know your limits. And that we won't prescribe medicines for which we are not authorised." (Nurse specialist 7)

\subsection{Difficulties surrounding nurse specialists' prescriptive authority and new professional power relations}

Even though almost all medical specialists said they had confidence in nurse specialists' prescribing practices and were satisfied with it, and although nurse specialists are legally responsible for their acts, including prescribing, medical specialists still felt that they had 'final responsibility' for both the nurse specialist and the patient. Some of them said they had difficulties with letting go of this responsibility, partly because they felt they might lose sight of their patients somewhat. Partly as a result of this, some nurse specialists and medical specialists 
Kroezen, M., Mistiaen, P., Dijk, L. van, Groenewegen, P.P., Francke, A.L. Negotiating jurisdiction in the workplace: a multiple-case study of nurse prescribing in hospital settings. Social Science)

\& Medicine: 2014, 117(sept), 107-115

still worked in a fairly strict supervision relationship. Medical specialists in particular emphasised the importance of this:

"My role is supervisory and actually the source of information for her, whereby, as time goes on, the information function will become more important than the monitoring task.” (Medical specialist 10)

"Also a little educational, supervising. You are and always will be working in some kind of training situation.” (Medical specialist 3)

A minority of the medical specialists questioned the added value of nurse specialists' prescriptive authority and a few reported negative experiences with prescribing by nurse specialists. Some medical specialists were sorry that task substitution in general made their professional practice more 'business-like', while others doubted its meaningfulness:

“(..) I have my doubts. Not about whether our nurse specialists are good or whatever, but I have never seen the added value being expressed in numbers and compared to the situation before." (Medical specialist 11)

A few medical specialists were downright negative about some aspects of nurse specialists' prescribing practices. This was mostly due to negative experiences with prescribing by nurse specialists.

"For example, sometimes medicines are prescribed where I think: it would have been better if you had not done that yet, or you could have waited with that a bit longer." (Medical specialist 6)

Overall, few of the nurse specialists in our sample said they personally had negative experiences with doctors who felt that their position was threatened by nurse specialists' prescriptive authority and none of the medical specialists in our sample said they saw it as a threat to their position. However, many of our respondents, whether nurse specialists or medical specialists, knew doctors who were negative about nurse specialists' prescriptive authority. Although this occurred amongst all age groups, it was repeatedly mentioned that younger medical specialists and medical specialist registrars or residents (doctors who are receiving advanced training in a specialist field of medicine) in particular felt threatened by nurse specialists:

"Let me put it differently, I think that the young generation of specialists who are coming up will think: 'those are chairs that we would have liked to sit on.'” (Medical specialist 11)

\subsection{Institutionalisation of nurse specialists' prescriptive authority}

On most hospital wards, nurse specialists' prescriptive authority had not yet been fully institutionalised. Most nurse specialists were still waiting for one or two minor organisational issues to be dealt with in order to complete their prescriptive authority, for example obtaining personal prescription paper.

“That approval of the list of medicines. I'm thinking: 'well, that's peanuts'. But it needs to be properly approved by all four medical specialists, and it has already taken me quite some time to get that done.” (Nurse specialist 11)

Despite the fact that these organisational issues had not yet been fully dealt with, nurse specialists were already prescribing as if the issues had been tackled.

An important organisational aspect is nurse specialists' possession of their own individual General Data Management-code (Algemeen GegevensBeheer-code or AGB-code in Dutch). This code can be assigned to individual healthcare professionals, practices and institutions. The code is listed on medical bills and is 
Kroezen, M., Mistiaen, P., Dijk, L. van, Groenewegen, P.P., Francke, A.L. Negotiating jurisdiction in the workplace: a multiple-case study of nurse prescribing in hospital settings. Social Science

\& Medicine: 2014, 117(sept), 107-115

used within the cost claims process in which the healthcare provider informs the health insurer of the care provided, so that the insurer can apply the appropriate rate when reimbursing healthcare expenditure. Only one nurse specialist already possessed her own AGB-code, some nurse specialists had requested an individual AGB-code, but the majority had not yet done so. Moreover, there was a remarkable degree of confusion about the AGB-code and its function in healthcare practice, both among nurse specialists and among medical specialists:

"I have heard that we can request such a code as nurse specialists, but I also heard that it is not necessary or worthwhile because the hospital already has some sort of code. It is unclear to me.” (Nurse specialist 3)

Another problematic issue that was repeatedly brought up by respondents was related to the so-called Diagnosis Treatment Combinations (Diagnose Behandel

Combinaties or DBCs in Dutch; the Dutch version of Diagnosis Related Groups). A DBC defines all the activities that are performed for the patient's diagnosis and treatment and the associated costs, and is used as a standardised classification of the care provided in the cost claims procedure. Since only medical specialists are allowed to open a DBC for a patient, their rates are used in cost claims, even though the actual patient care may have been provided by nurse specialists.

\section{DISCUSSION}

\subsection{Prescribing by nurse specialists in the workplace}

Across hospitals and hospital wards, we found a great variety in both the extent to which and way in which nurse specialists' legal prescriptive authority had been implemented. This already became apparent during our inclusion process, as it turned out that nurse specialists in some hospitals were not yet prescribing medicines, even though they had the legal authority to do so. Among our final sample of prescribing nurse specialists, there was considerable variety in the number and range of medicines prescribed. Moreover, whilst prescribing, nurse specialists used a wide variety of supporting documents with different levels of applicability, ranging from guidelines drafted by international professional associations to personal formularies developed by the individual nurse specialist. Yet the manner in which the prescribing process took place was fairly similar for all prescribing nurse specialists; they were very much aware of their limitations when it came to prescribing and regularly consulted medical specialists about their prescribing decisions, almost always in an informal way. Both nurse specialists and medical specialists reported that they liked working in this way.

It may seem somewhat surprising that on the work floor, nurse specialists and medical specialists generally cooperate in a harmonious way. Especially considering the struggles that took place at macro level between medical associations and nursing associations regarding jurisdiction over prescribing in the Netherlands, and as is usually the case internationally (Ball, 2009, Jones, 1999, Kroezen et al., 2012a, Kroezen et al., 2012b, McCann and Baker, 2002, Nilsson, 1994, Plonczynski et al., 2003 and Romero-Collado et al., 2013). In the sociology of professions, including Abbott's framework (1988), the emphasis is often on active and overt opposition from professions to defend their professional jurisdiction, as was can be found at macro level for nurse prescribing. However, our results show that professional opposition does not always have to be overt, at least not at micro level. Professions can take a cooperative stance and whilst doing so make sure that legal rules are being 
negotiated and framed in such a way that they retain the (amount of) professional

jurisdiction they want. A second thing that should be taken into account is that once physicians have experience with nurse prescribing, their views become more positive than when they lack this hands-on experience (Latter et al., 2010). This can also explain the discrepancy between the quite fierce negotiations taking place at macro level and the more harmonious negotiations taking place at on the work floor.

\subsection{Division of jurisdictional control over prescribing between nurse specialists and physicians in the workplace}

From a macro point of view, the division with regard to prescribing is unambiguous. We already noted that in the legal arena, nurse specialists and physicians share highly comparable full jurisdiction over prescribing. Yet due to the great variety in the manner in which this legal framework is currently being implemented and drawn up in the workplace, at the micro level a variety of jurisdictional settlements coexists concerning nurse specialist prescribing. We found that nurse specialists hardly ever independently prescribe all medicines within their specialism and competence, as their legal authority allows them to do. Most of the time, medical specialists, consciously or unconsciously, still play a large role in nurse specialists' prescribing processes, thereby maintaining a situation of jurisdictional nursing subordination. Sometimes medical specialists drafted strict prescribing protocols for nurse specialists, so that the intellectual jurisdiction over prescribing remained with the medical profession (Abbott, 1988). So even though nurse specialists were allowed to prescribe, medical specialists retained control over the cognitive content of nurse specialists' prescribing practices. This is in line with findings by Currie et al. (2012) who showed that clinical genetics defined the limits of new policy-driven nurse roles by utilizing stipulated guidelines. We also found that nurse specialists were allowed to prescribe for 'routine patients', while the more complex patients were exclusively treated by the medical specialist. Abbott (1988) would label this as client differentiation, as the two professional groups serve different patient groups. By restricting nurse specialists' prescribing practices to 'routine patients', medical professionals kept the professionally 'higher status' patients for themselves. In terms of professional dominance, one could even say that the introduction of nurse specialist prescriptive authority enabled medical specialists to assign professionally 'dangerous' routine work to nurse specialists and, in so doing, enhance their professional position and reinforce the subordinate position of nursing (Abbott, 1988). Hence, in line with other studies on enacted professional jurisdictions (Currie et al., 2012), medical professionals seem to shape new extended nursing roles in such a way as to ensure their continued professional dominance.

\subsection{Workplace jurisdiction and legal jurisdiction over prescribing}

As said before, there is a considerable discrepancy between the division of jurisdictional control over prescribing at the macro (legal) level and at the micro (workplace) level. As found earlier, legal jurisdiction seems to have a rather vague relation to professional workplace realities (Abbott, 1988, Sanders and Harrison, 2008 and Spilsbury and Meyer, 2004).

Allen (2000) and Currie et al. (2010) noted that features of the work setting, such as the recognition (or lack of recognition) of new sets of knowledge and skills in work roles, mediate the legal division of labour. In the case of nurse specialists' prescriptive authority, we found that hospitals, medical boards and/or nurse 
specialists applied additional rules and limitations to nurse specialists' legal authority to independently prescribe medicines. Moreover, nurse specialists, on their own initiative, often consulted with their medical specialist before or after prescribing a medicine. This is understandable, given that nurse specialists obtained prescriptive authority only recently and may feel they are in a learning process. Yet this limited their broad legal prescriptive authority in everyday practice to a much narrower jurisdiction. However, it remains unclear what the exact mechanisms and factors are that explain the large variety of jurisdictional settlements found in the workplace when it comes to nurse specialists' prescriptive authority. Because the introduction of prescriptive authority for nurse specialists can be looked upon as a service reform, explanatory factors could, for example, be sought in differences in work culture, network organisation and leadership styles among the different hospitals and wards (Martin et al., 2009). These meso-level factors should be taken into account for a broader understanding about the discrepancy between macro- and micro level when it comes to jurisdiction over prescribing. It may for example be possible that within relatively small wards, where strong ties exist between nurse specialists and medical specialists, medical specialists may hand over tasks more easily than within large hierarchy-based wards, where ties between the different healthcare professionals may be much looser and job roles and task descriptions are more formalised, i.e. restricted, as a result. This would be in line with our findings that years of accumulated cooperation and trust between nurse specialists and medical specialists appeared to result in good cooperation where the prescribing of medicines was concerned. However, this also raises the question of how sustainable this source of good cooperation, i.e. trust, will turn out to be in the future. After all, if prescribing nurse specialists start to change jobs, medical specialists will have to cooperate with nurse specialists who they hardly know and hence have no 'trust relationship' with. The great variety in the extent to which and way in which nurse specialists' legal prescriptive authority has been implemented across hospitals and hospital wards is somewhat remarkable. It means that "prescribing by nurse specialists", as spoken about by policymakers, healthcare professionals and patients alike, is nothing more than an umbrella term. Two people could be using the same expression while having a significantly different reality in mind. This may hamper the professionalisation of the nursing profession. After all, it is the knowledge that a profession possesses which defines the profession, as stated by Abbott (1988). If the knowledge level of the profession is ambiguous, as it is in terms of what is understood by nurse prescribing, the status of the profession itself may become ambiguous.

The discrepancy that we found between the division of jurisdictional control over prescribing at the legal level and at the workplace level has implications for policy expectations as well. If, as our results suggest, nurse specialists in the workplace prescribe less often and in a less independent way than their legal authority allows them to do, expectations about the potential impact of nurse specialist prescribing on healthcare may need to be adjusted. The positive effects of prescribing by nurse specialists, for example in terms of cost efficiency, may be lower than expected and/or hoped for. Moreover, the prescribing of medicines is by no means the only task substitution that is taking place within nursing. Task substitution is increasingly seen as a solution to current problems in health care. Our study shows that the implications and effects of task substitutions should not only be studied and discussed at legal macro level, but also at organisational level. However, prescribing 
is a relatively new task for Dutch nurse specialists. It is quite possible that nurse specialists and medical specialists need to go through a habituation period. Perhaps in the future, nurse specialists will start prescribing more frequently and for a wider range of medicines, doing so in a more independent way. Simultaneously, the variety across hospitals and wards found shortly after the introduction of nurse specialists' prescriptive authority may diminish as well and a more 'common practice' may develop.

Finally, attention should be paid to the financial organisation of nurse prescribing. Our results suggest that in the Netherlands, the financing structure of nurse specialists' prescriptions is opaque and confusing. Almost all nurse specialists in our study prescribed under the General DataManagement-code (Algemeen GegevensBeheer-code or AGB-code in Dutch) of their medical specialist. Hence, their prescriptions are untraceable for insurers and policymakers in the financial systems, making it impossible to evaluate nurse specialists' prescriptive authority on a cost-efficiency basis. Yet cost efficiency was precisely one of the main reasons for introducing nurse specialists' prescriptive authority ( Dutch House of Representatives, 2011, Kroezen et al., 2011, Kroezen et al., 2012a, Kroezen et al., 2012b and Ministry of Health, 2011). Internationally, there is a need for detailed evaluations of the cost effectiveness of nurse prescribing as well ( Courtenay and Carey, 2006, Latter and Courtenay, 2004 and Sibbald et al., 2004). However, this is only one possible criterion for the evaluation of nurse specialist prescribing. Internationally, it has been shown, for example, that patients are generally more satisfied with prescribing by nurses or nurse specialists compared with physicians ( Van Ruth et al., 2008) and that nurses obtain higher medication adherence rates with patients compared with medical specialists ( Andersson et al., 2005). These are important aspects in their own right which may indirectly also lead to increased cost efficiency.

\subsection{Limitations}

Several limitations of the study bear mentioning. It is likely that our sample of prescribing nurse specialists is not representative for all nurse specialists in the Netherlands. After all, the nurse specialists who participated in our study were already prescribing medicines and can be considered highly motivated early adapters in that regard. Hence, prescribing may be less developed among other hospitals and/or nurse specialists and prescribing might take place in a less independent way. Moreover, the nurse specialists who participated in our study proposed the medical specialists we approached for interviewing. Again, this may have created a biased subsample, as it is likely that nurse specialists selected medical specialists with whom they have a good cooperative relationship. Nonetheless, our study adds to the relatively small body of research studying the division of jurisdictional control at the micro or workplace level (Bechky, 2003, Sanders and Harrison, 2008 and Spilsbury and Meyer, 2004). By uncovering the discrepancy between nurse specialists' legal jurisdiction over prescribing and their jurisdiction in the work arena, it shows that professional negotiations over jurisdiction take place at both the macro level and the micro levels, with different divisions of jurisdictional control as a result.

\section{ACKNOWLEDGMENTS}

The authors wish to thank Janneke Dekker MSc and Sanne Gielen MSc for their help in collecting the data. We would also like to thank all the participating nurse 
Kroezen, M., Mistiaen, P., Dijk, L. van, Groenewegen, P.P., Francke, A.L. Negotiating jurisdiction in the workplace: a multiple-case study of nurse prescribing in hospital settings. Social Science) \& Medicine: 2014, 117(sept), 107-115

specialists and medical specialists as well as all the patients who contributed to the study by allowing us to sit in on their consultations with the nurse specialists.

\section{REFERENCES}

Abbott, 1988A. AbbottThe System of Professions: an Essay on the Division of Expert LaborThe University of Chicago Press, Chicago (1988)

Allen, 1996D. AllenThe Shape of General Hospital Nursing: the Division of Labour at WorkUniversity of Nottingham (1996)

Allen, 1997D. AllenThe nursing-medical boundary: a negotiated order?Sociol. Health \& IIIn., 19 (1997), pp. 498-520

Allen, 2000D. AllenDoing occupational demarcation the boundary-work of nurse managers in a district general hospitalJ. Contemp. Ethnogr., 29 (3) (2000), pp. 326-356

Andersson et al., 2005K. Andersson, A. Melander, C. Svensson, O. Lind, J.L. NilssonRepeat prescriptions: refill adherence in relation to patient and prescriber characteristics, reimbursement level and type of medicationEur. J. Public Health, 15 (6) (2005), pp. 621626

Ball, 2009J. BallImplementing Nurse Prescribing: an Updated Review of Current Practice InternationallyInternational Council of Nurses, Geneve (2009)

Barley, 1986S.R. BarleyTechnology as an occasion for structuring: evidence from observations of CT scanners and the social order of radiology departmentsAdm. Sci. Q. (1986), pp. 78-108

Barrett et al., 2012M. Barrett, E. Oborn, W.J. Orlikowski, J. YatesReconfiguring boundary relations: robotic innovations in pharmacy workOrgan. Sci., 23 (5) (2012), pp. 1448-1466

Bechky, 2003B.A. BechkyObject lessons: workplace artifacts as representations of occupational jurisdictionAm. J. Sociol., 109 (2003), pp. 720-752

Buchan and Dal Poz, 2002J. Buchan, M.R. Dal PozSkill mix in the health care workforce: reviewing the evidenceBull. World Health Organ., 80 (7) (2002), pp. 575-580

Buckley et al., 2006P. Buckley, J. Grime, A. Blenkinsopplnter- and intra- professional perspectives on non-medical prescribing in an NHS trustPharm. J., 277 (2006), pp. 394398

Courtenay and Carey, 2006M. Courtenay, N. CareyNurse-led care in dermatology: a review of the literatureBr. J. Dermatol., 154 (1) (2006), pp. 1-6

Courtenay and Carey, 2009M. Courtenay, N. CareyNurse prescribing by children's nurses: views of doctors and clinical leads in one specialist children's hospitalJ. Clin. Nurs., 18 (18) (2009), pp. 2668-2675

Currie et al., 2010G. Currie, R. Finn, G. MartinRole transition and the interaction of relational and social identity: new nursing roles in the English NHSOrgan. Stud., 31 (7) (2010), pp. 941-961

Currie et al., 2012G. Currie, A. Lockett, R. Finn, G. Martin, J. WaringInstitutional work to maintain professional power: recreating the model of medical professionalismOrgan. Stud., 33 (7) (2012), pp. 937-962

Dutch House of Representatives, 2011Dutch House of RepresentativesTweede Kamer der Staten-Generaal, vergaderjaar 2010-2011, 32 196, 32 261, nr. 13, Brief van de Minister van Volksgezondheid, Welzijn en Sport [The Dutch House of Representatives, year 20102011, 32 196, 32 261, no. 13, Letter of the Minister of Health, Welfare and Sport] (2011) (Den Haag)

Fisher, 2010R. FisherNurse prescribing: a vehicle for improved collaboration, or a stumbling block to inter-professional working?Int. J. Nurs. Pract., 16 (2010), pp. 579-585

Gerrish et al., 2003K. Gerrish, M. McManus, P. AshworthCreating what sort of professional? Master's level nurse education as a professionalising strategyNurs. Inq., 10 (2) (2003), pp. 103-112

Goundrey-Smith, 2008S. Goundrey-SmithElectronic medicines management and nonmedical prescribingK.J. Hannah, M.J. Ball (Eds.), Principles of Electronic Prescribing, Springer-Verlag, London (2008), pp. 119-136

Green and Thorogood, 2004J. Green, N. ThorogoodQualitative Methods for Health ResearchSAGE Publications Ltd, London (2004) 
Kroezen, M., Mistiaen, P., Dijk, L. van, Groenewegen, P.P., Francke, A.L. Negotiating jurisdiction in the workplace: a multiple-case study of nurse prescribing in hospital settings. Social Science \& Medicine: 2014, 117(sept), 107-115

Horrocks et al., 2002S. Horrocks, E. Anderson, C. SalisburyDo nurse practitioners working in primary care provide equivalent care to doctors?BMJ, 324 (2002), pp. 819-823

Jones, 1999M. JonesNurse Prescribing: Politics to PracticeBailliere Tindall \& Royal College of Nursing, Edinburgh (1999)

Kroezen et al., 2012aM. Kroezen, A.L. Francke, P.P. Groenewegen, L. Van DijkVoorschrijfbevoegdheid voor verpleegkundigen: visies en verwachtingen van Nederlandse stakeholders [Nurse prescribing: views and expectations of Dutch stakeholders] Verpleegkunde, 2 (2012), pp. 10-16

Kroezen et al., 2011M. Kroezen, L. Van Dijk, A.L. Francke, P.P. GroenewegenNurse prescribing of medicines in Western European and Anglo-Saxon countries: a systematic review of the literatureBMC Health Serv. Res., 11 (127) (2011)

Kroezen et al., 2012bM. Kroezen, A.L. Francke, P.P. Groenewegen, L. van DijkNurse prescribing of medicines in Western European and Anglo-Saxon countries: a survey on forces, conditions and jurisdictional controllnt. J. Nurs. Stud., 49 (8) (2012), pp. 1002-1012

Kroezen et al., 2013aM. Kroezen, L. van Dijk, P.P. Groenewegen, M. de Rond, A.J. de Veer, A.L. FranckeNeutral to positive views on the consequences of nurse prescribing: results of a national survey among registered nurses; nurse specialists and physiciansint. J. Nurs. Stud., 51 (4) (2013), pp. 539-548

Kroezen et al., 2013bM. Kroezen, L. van Dijk, P.P. Groenewegen, A.L. FranckeKnowledge claims, jurisdictional control and professional status: the case of nurse prescribing

PloS One, 8 (10) (2013), p. e77279

Latter and Courtenay, 2004S. Latter, M. CourtenayEffectiveness of nurse prescribing: a review of the literatureJ. Clin. Nurs., 13 (1) (2004), pp. 26-32

Latter et al., 2010S. Latter, A. Blenkinsopp, A. Smith, S. Chapman, M. Tinelli, K. Gerard, P. Little, N. Celino, T. Granby, P. NichollsEvaluation of Nurse and Pharmacist Independent PrescribingUniversity of Southampton; Keele University (2010)

Lewis, 2001R. LewisNurse-led Primary Care: Learning from PMS PilotsKing's Fund Publishing, London (2001)

Martin et al., 2009G.P. Martin, G. Currie, R. FinnLeadership, service reform, and publicservice networks: the case of cancer-genetics pilots in the English NHSJ. Public Adm. Res. Theory, 19 (4) (2009), pp. 769-794

MAXQDA, 2007MAXQDAVERBI SoftwareConsult.GmbH, Marburg, Germany (2007)

McCann and Baker, 2002T.V. McCann, H. BakerCommunity mental health nurses and authority to prescribe medications: the way forward?J. Psychiatr. Ment. Health. Nurs., 9 (2002), pp. 175-182

Mclaughlin and Webster, 1998J. Mclaughlin, A. WebsterRationalising knowledge: IT systems, professional identities and powerSociol. Rev., 46 (4) (1998), pp. 781-802Ministry of Health, 2011Ministry of Health, W.a.SBesluit van 21 december 2011, houdende tijdelijke regels inzake de zelfstandige bevoegdheid tot het verrichten van voorbehouden handelingen van verpleegkundig specialisten (Tijdelijk besluit zelfstandige bevoegdheid verpleegkundig specialisten) [Decision of 21 December, on temporary rules relating to the autonomous power to perform restricted actions of nurse specialists (Temporary autonomous decision power nurse specialists)] Staatsbl. het Koninkr. Ned., 659 (2011) Mizrachi and Shuval, 2005N. Mizrachi, J.T. ShuvalBetween formal and enacted policy: changing the contours of boundariesSoc. Sci. Med., 60 (7) (2005), pp. 1649-1660

Nilsson, 1994M. NilssonOpposition to nurse-prescribing in SwedenLancet, 1077 (1994)

Petrakaki et al., 2012D. Petrakaki, N. Barber, J. WaringThe possibilities of technology in shaping healthcare professionals:(Re/De-) professionalisation of pharmacists in EnglandSoc. Sci. Med., 75 (2) (2012), pp. 429-437

Plonczynski et al., 2003D. Plonczynski, N. Oldenburg, M. BuckThe past, present and future of nurse prescribing in the United StatesNurse Prescr., 1 (4) (2003), p. 170

Polit and Beck, 2004D.F. Polit, C.T. BeckNursing Research: Principles and

MethodsLippinott, Williams \& Wilkins, Philadelphia (2004)

Raad voor de Volksgezondheid en Zorg, 2002Raad voor de Volksgezondheid en ZorgTaakherschikking in de gezondheidszorgRVZ, Zoetermeer (2002)

Romero-Collado et al., 2013A. Romero-Collado, E. Homs-Romero, E. Zabaleta-del-Olmo, D. Juvinya-CanalNurse prescribing in primary care in Spain: legal framework, historical 
Kroezen, M., Mistiaen, P., Dijk, L. van, Groenewegen, P.P., Francke, A.L. Negotiating jurisdiction in the workplace: a multiple-case study of nurse prescribing in hospital settings. Social Science

\& Medicine: 2014, 117(sept), 107-115

characteristics and relationship to perceived professional identityJ. Nurs. Manag., 22 (2013), pp. 394-404

Salhani and Coulter, 2009D. Salhani, I. CoulterThe politics of interprofessional working and the struggle for professional autonomy in nursingSoc. Sci. Med., 68 (7) (2009), pp. 12211228

Sanders and Harrison, 2008T. Sanders, S. HarrisonProfessional legitimacy claims in the multidisciplinary workplace: the case of heart failure careSociol. Health \& IIIn., 30 (2) (2008), pp. 289-308

Sibbald et al., 2004B. Sibbald, J. Shen, A. McBrideChanging the skill-mix of the health care workforceJ. Health Serv. Res. Policy, 9 (Suppl. 1) (2004), pp. 28-38

Snelgrove and Hughes, 2000S. Snelgrove, D. HughesInterprofessional relations between doctors and nurses: perspectives from South WalesJ. Adv. Nurs., 31 (2000), pp. 661-667

Spilsbury and Meyer, 2004K. Spilsbury, J. MeyerUse, misuse and non-use of health care assistants: understanding the work of health care assistants in a hospital settingJ. Nurs. Manag., 12 (6) (2004), pp. 411-418

Van der Peet, 2010R. Van der PeetDe zelfstandige bevoegdheid van de verpleegkundig specialist [The independent authority of the nurse specialist] Tijdschr. Voor Verpleegkundigen, 120 (7/8) (2010), pp. 45-49

Van Meersbergen, 2011D.Y.A. Van MeersbergenTask shifting in the NetherlandsWorld Med. J., 57 (4) (2011), pp. 126-130

Van Ruth et al., 2008L. Van Ruth, A.L. Francke, P. MistiaenEffects of nurse prescribing of medication: a systematic reviewInternet J. Healthc. Adm., 5 (2) (2008)

Verpleegkundigen \& Verzorgenden Nederland, 2013

Verpleegkundigen \& Verzorgenden Nederland, 2013. Verpleegkundigspecialismen.nl.: 18.11.13.).

Wanless, 2002D. WanlessSecuring Our Future Health: Taking a Longterm ViewThe Public Enquiry Unit, London (2002) 
Kroezen, M., Mistiaen, P., Dijk, L. van, Groenewegen, P.P., Francke, A.L. Negotiating jurisdiction in the workplace: a multiple-case study of nurse prescribing in hospital settings. Social Science

TABLES AND BOXES

\section{Box 1}

Nurse specialists in The Netherlands.

Nurse specialists are registered nurses who have successfullycompleted a two-year Master's programme in Advanced Nursing Practice and have subsequently registered themselves in the Nurse Specialists Register (Verpleegkundig Specialisten Register in Dutch; (Verpleegkundigen \& Verzorgenden Nederland, 2013). There are five nurse specialisms in the Netherlands, namely acute care, chronic care, intensive care, preventive careand mental health care (Dutch House of Representatives, 2011; Ministry of Health, 2011). Nurse specialists work autonomously at the interface between medical and nursing care, and treat defined groups of patients with whom they establish an individual care relationship. Since January 2012, they have been allowed to prescribe any licensed medicine for any medical condition within their specialism and competence. However, their prescriptive authority is part of the so-called 'experimental article' (Article 36a) in the Dutch Individual Healthcare Professions Act (Wet BIG in Dutch). This means that nurse specialists are allowed to perform reserved procedures, including the prescribing of medicines, for a trial period of five years. If this experiment is evaluated as having been a success, a final arrangement may be included in the law, granting nurse specialists permanent authority to perform reserved procedures, including prescribing. Apart from the legal framework provided by the government, there has been limited official support for healthcare organisations and/or individual nurse specialists on how to translate nurse specialists' prescriptive authority in everyday work practices. One important guide that has been developed in this regard is the 'Guide to the implementation of task substitution' (Handreiking implementatie taakherschikking in Dutch), jointly written by the Royal Dutch Medical Association (KNMG), the Dutch Nurses' Association (V\&VN) and the Netherlands Association of Physician Assistants (NAPA) (KNMG, V\&VN \& NAPA, 2012). 
Kroezen, M., Mistiaen, P., Dijk, L. van, Groenewegen, P.P., Francke, A.L. Negotiating jurisdiction in the workplace: a multiple-case study of nurse prescribing in hospital settings. Social Science \& Medicine: 2014, 117(sept), 107-115

\section{[TABLE 1]}

Specialisms of participating nurse specialists and medical specialists.

\begin{tabular}{|l||l|l||}
\hline \multicolumn{1}{|c|}{ Specialism } & Nurse specialists & Medical specialists \\
\hline \hline Breast cancer care & 2 & - \\
\hline \hline Diabetes care & 1 & 1 \\
\hline Haematology & 3 & 3 \\
\hline Lung diseases & 1 & 1 \\
\hline Nephrology & 3 & 3 \\
\hline Neonatology & 1 & 1 \\
\hline Oncology/palliative care & 2 & 3 \\
\hline Thoracic surgery & 1 & 1 \\
\hline Wound care & 1 & - \\
\hline \hline Vascular surgery & - & 1 \\
\hline Total & 15 & 14 \\
\hline \hline
\end{tabular}

\section{Box 2}

Topic guide for interviews with nurse specialists and medical specialists.

- Personal views on nurse specialists' prescriptive authority

- Prescribing training

- Prescribing experiences

- Considerations whilst prescribing

- Cooperation and relationship with medical specialist/ nurse specialist and other healthcare professionals where prescribing is concerned

- Organisational conditions

- Future expectations regarding prescribing 